\title{
IRREDUCIBLE HOMOGENEOUS LINEAR GROUPS IN AN
}

\author{
ARBITRARY DOMAIN* \\ BY

\section{WILLIANI BENJAMIN FITE}

I have given elsewhere a necessary and sufficient condition that certain categories of abstract groups of finite order be simply isomorphic with irreducible homogeneous linear groups in the domain of all real and complex numbers. $\dagger$ In the present paper I establish a two-fold generalization of this result by showing that the same condition applies to all groups of finite order and to an arbitrary domain.

The proof for the necessity of this condition rests upon a conclusion drawn from Theorem II of Schur's Neue Begrïndung der Theorie der Gruppencharaktere. +

Suppose that we have a homogeneous linear group $G$ of finite order whose coefficients belong to $\Omega$ (an arbitrary finite field or an arbitrary domain) and which is irreducible in $\Omega$. If $P$ is a given substitution on the same $f$ variables as $G$ with coefficients in $\Omega$, the equation

$$
\left|P-x E_{f}\right|=0
$$

is an equation in the unknown $x$ with known ceefficients. If this equation has a root in $\Omega$ and if $P$ is commutative with every substitution of $G$, ScHUR's proof of his 'Theorem II applies, therefore $P$ must be a similarity substitution.

If $\Omega$ is any finite field, say the $G F\left[p^{m}\right]$, then in the field $F_{p}$, which is the aggregate of all the fields $G F\left[p^{i}\right](i=1,2, \cdots), G$ can be so transformed that in the matrix of every substitution of its central $H$ all the elements below the main diagonal are zero. If $\Omega$ is a domain, the same result can be accom-

* Presented to the Society, in a somewhat different form, December $31,1908$.

†Transactions of the American Mathematical Society, vol. 7 (1906), p. 65 ; Bulletin of the American Mathematical Society, vol. 14 (1908), p. 32t.

$\ddagger$ Berliner sitzungsberichte, 1905, I, p. 410.

?.Cf. Dickson, Transactions of the American Mathematical Society, vol. 8 (1907), p. 389; Bulletin of the American Mathematical Society, rol. 13 (1907), p. 477 . 
plished by passing to the domain of all real and complex numbers.* In this transformed form of $G$ no two substitutions $S_{1}$ and $S_{2}$ of $H$ can change $x_{j}$ into the same function, since, if this were possible, $S_{1} S_{2}^{-1}$ would leave $x_{j}$ unchanged - that is, one of the multipliers of $S_{1} S_{2}^{-1}$ would be unity. But, since this substitution is invariant in the irreducible group $G$, our conclusion from Schur's Theorem shows that this is possible only in case $S_{1} S_{2}^{-1}$ is a similarity substitution with its multipliers all equal to unity - that is, in case $S_{1}^{r} S_{2}^{-1}$ is the identical substitution. But $S_{1}$ and $S_{2}$ were taken as distinct substitutions.

If now $I I$ is of order $p_{1}^{a_{1}} p_{2}^{a_{2}} \cdots p_{r}^{a_{r}}$, where $p_{1}, p_{2}, \cdots, p_{r}$, are distinct primes, it is the direct product of groups $H_{1}, I H_{2}, \ldots, I I_{r}$, of order $p_{1}^{\alpha_{1}}, p_{2}^{\alpha_{2}}, \cdots, p_{r}^{\alpha_{r}}$, respectively; and it follows from what has just been proved that $I I_{i}(i=1,2, \ldots, v)$, can contain only one subgroup of order $p_{i}$ and is therefore cyclic. $\dagger$ IIence II is cyclic.

Suppose now that $G$ is any group of finite order $g$ with a cyclic central generated by the operation $h$ of order $a(>1)$. If we write $G$ as a regular permutation group $h$ will take the form

$$
h \equiv\left(x_{11} x_{12} \cdots x_{1 a}\right)\left(x_{21} x_{22} \cdots x_{2 u}\right) \cdots\left(x_{\gamma 1} x_{\gamma 2} \cdots x_{\gamma a}\right),
$$

where $\gamma=g / a$. If now we write this permutation group as a homogeneous linear group and then transform it by the substitution

$$
S: \quad y_{i j}=\sum_{k=1}^{a} \omega^{(k-1)(j-1)} x_{i k},
$$

where $\omega$ is a primitive $a$ th root of unity, $h$ will be transformed into its normal form. Moreover in this transformed form of $G$ the variables

$$
x_{1 j}, x_{2 j}, \cdots, x_{\gamma j} \quad(j=1,2, \cdots, a)
$$

are transformed among themselves in semi-canonical form. $\neq$ We shall denote the group formed by the substitutions on these variables by $G_{j}$. If $j-1$ is relatively prime to $a, G_{j}$ is simply isomorphic with $G$.

If $G$ were not simply isomorphic with any irreducible group in $C$ (the domain of all real and complex numbers), then when $G_{j}(j-1$ relatively prime to $\alpha)$ is completely reduced in $C$ there must be some irreducible component such that when all the irreducible representations of $G$ that are equivalent to this

* Cf. I Rber, Algebra, II (second edition), pp. 172, 173; 176-178. This discussion of WEBER's is with reference to the domain of all real and complex numbers, but the processes described are valid in the field $F_{\mu}$.

† BuRsside, Theory of groups of finite oriler, pp. 73,75 . If $p_{i}=2, H_{i}$ could not be the noncyclic group with only one subgroup of order 2 , since this contains more than one cyclic subgroup of order 4.

†Cf. Transactions of the American II athematical Society, rol. 8 (1907), p. 108. 
one (in number equal to the degree of each one*) are taken together, there is an $r_{1}, r_{2}$ isomorphism between the group formed by these representations and the group in the remaining variables, and $r_{1}>1, r_{2}>1$; since otherwise $G$ would be simply isomorphic with some irreducible group. Call these two components $L_{1}$ and $L_{2}$ respectively and let their orders be denoted by $l_{1}$ and $l_{2}$ respectively. If $H_{i}(i=1,2)$ is the subgroup of $L_{i}$ that corresponds to identity in the other component, $H_{i}$ can contain no invariant substitution of $L_{i}$, except identity, since every invariant substitution $G_{j}$ is a similarity substitution. Now each irreducible component of $L_{1}$ is of degree at most $\sqrt{l_{1} / a}$, since each such component is of order $l_{1}$ and contains at least $a$ invariant substitutions. $\dagger$ But by virtue of the manner in which $G_{j}$ is formed from $L_{1}$ and $L_{2}$ we must have $g=r_{1} r_{2} \cdot l_{1} / r_{1}$ and $l_{1}=g / r_{2}$. Then the number of variables in $L_{1}$ is at most $g /\left(a r_{2}\right)$.

Any substitution of $H_{1}$, such as $t$ of order $b$, when combined with identity of $H_{2}$ gives a substitution of $G_{j}$ that has at least $g / a-g /\left(a r_{2}\right)$ multipliers equal to unity. Now there are $\phi(a)$ values of $j$ such that $j-1$ is relatively prime to $a$ and there is a similar substitution in each $G_{j}$ corresponding to these values of $j$. These similar substitutions combine with one another and with a certain substitution from every $G$ ( $j-1$ not relatively prime to $a$ ) to form a certain substitution of $G$ of order $b$.

Since every $G_{j}$ is in semi-canonical form, in any substitution of $G_{j}$ the variables are permuted among themselves (except for certain coefficients). If now $j-1$ is not relatively prime to $a$, the cycles of the permutation in a substitution of $G_{j}$ that corresponds to a non-invariant substitution of $G$ must all be of the same order, since otherwise a certain power of this substitution that is not invariant in $G$ would change the variables of at least one of the sets of variables into themselves multiplied by certain constants. This means that in the regular permutation group there would be a non-invariant operation that leaves some of the systems of intransitivity of $H$ unchanged. But this is impossible in a regular group. Hence in $G_{j}(j-1$ not relatively prime to $a)$ the variables of $t_{j}$ (the substitution that corresponds to $t$ ) are permuted among themselves (except for certain coefficients) in cycles of order $b$. The characteristic determinant $D_{j}$ of $t_{j}$ is then the product of $g / a b$ determinants each of order $b$ :

$$
D_{j} \equiv\left\{(-1)^{b} \lambda^{b}-(-1)^{b}\right\}^{g / a b} .
$$

Hence $t_{j}$ has $g / a b$ multipliers equal to unity. The total number of multipliers that equal unity in the substitution of $G$ that corresponds to $t$ would then be at least

$$
\left(\frac{g}{a}-\frac{g}{a r_{2}}\right) \phi(a)+\frac{g}{a b}[a-\phi(a)] .
$$

* Burnside, Acta Mathematica, vol. 28 (1904), p. 383.

†Transactions of the American Mathematical Sooiety, vol. 7 (1906), p. 67. 
But this number is greater than the actual number of multipliers of this substitution that equal unity, namely* $g / b$, provided $r_{2}>2$. However, if $r_{2}=2$, $H_{2}$ would contain only one substitution hesides identity. This substitution would be invariant in $L_{2}$ and would combine with identity of $L_{1}$ to form an invariant substitution of $G_{j}$ that is not a similarity substitution.

Hence $G$ must be simply isomorphic with an irreducible group in $C$.

If $G$ has no invariant operation except identity. we can form the lirect product $\bar{G}$ of $G$ and an operation $h$ of order $\iota(>1)$. It follows from the preceding discussion that $\bar{G}$ is simply isomorphic with an irreducible group in $C^{\prime}$. The subgroup of this irreducible group that corresponds to $G$ must also be irreducible, since otherwise, in view of the fact that the substitution corresponding to $h$ is a similarity substitution, the group that is simply isomorphic with $\bar{G}$ would be reducible.

Hence any group of finite order with a cyclic central is simply isomorphic with a homogeneous linear group that is irreducible in $C^{\prime}$. But every irreducible representation of $G$ (in $C$ ) is equivalent to one of the irreducible components of the regular permutation group that is simply isomorphic with $G$ when this is written as a homogeneous linear group. $t$ If now no irreducible component of this latter group in any domain $\Omega$ were simply isomorphic with $G$, then no irreducible component in $C$ could be simply isomorphic with $G$.

We can formulate the results of the precerling discussion into the

Thвorkar. A nccessary and sufficient comdition that any group of finite srder be simply isomorphic with an irreducible group in an!y domain is that its central be cyclic.

It should be remarked that the sufficiency of this condition is established only for an arbitrary domain, whereas the necessity of it is established also for any finite field.

Corsell UNiversity.

*Trausactions of the American Mathematical Society, vol. 8 (1907), p. 108.

† BL:Rside, loc. cit., p. 387. 